\title{
RECENT PROGRESS IN THE REGULARITY THEORY OF FOURIER INTEGRALS WITH REAL AND COMPLEX PHASES AND SOLUTIONS TO PARTIAL DIFFERENTIAL EQUATIONS
}

\author{
MICHAEL RUZHANSKY \\ Department of Mathematics, Imperial College \\ London SW7 2BZ, United Kingdom \\ E-mail:ruzh@ic.ac.uk
}

\begin{abstract}
In this paper we will give a brief survey of recent regularity results for Fourier integral operators with complex phases. This will include the case of real phase functions. Applications include hyperbolic partial differential equations as well as non-hyperbolic classes of equations. An application to an oblique derivative problem is also given.
\end{abstract}

1. Introduction. Let us start with the definition of a Fourier integral operator with a complex phase. Informally, it is an operator $T$ that can be locally written in the form

$$
T u(x)=\int_{\mathbf{R}^{n}} \int_{\mathbf{R}^{N}} e^{i \Phi(x, y, \theta)} a(x, y, \theta) u(y) d \theta d y,
$$

with a symbol $a$ and a complex valued phase function $\Phi$ with $\operatorname{Im} \Phi \geq 0$.

Let us give a more rigorous definition now. Let $V$ be a conic subset of $\mathbf{R}^{n} \times \mathbf{R}^{n} \times$ $\left(\mathbf{R}^{N} \backslash\{0\}\right):(x, y, \theta) \in V$ implies $(x, y, \lambda \theta) \in V$ for all $\lambda>0$. A smooth in $V$ function $\Phi=\Phi(x, y, \theta)$ is called a regular phase function of positive type if

(i) $\Phi$ has no critical points in $V: d_{(x, y, \theta)} \Phi \neq 0$ in $V$.

(ii) $\Phi$ is positive homogeneous in $\theta$ : $\Phi(x, y, \lambda \theta)=\lambda \Phi(x, y, \theta)$ for all $\lambda>0$.

(iii) $d\left(\partial_{\theta_{1}} \Phi\right), \ldots, d\left(\partial_{\theta_{n}} \Phi\right)$ are linearly independent over $\mathbf{C}$ on $C_{\Phi \mathbf{R}}=\{(x, y, \theta)$ : $\left.\partial_{\theta} \Phi=0\right\}$.

(iv) $\operatorname{Im} \Phi(x, y, \theta) \geq 0$ on $V$.

Let us immediately note that by the equivalence of phase function theorem $([8],[5])$ we can rewrite general Fourier integral operators microlocally with $N=n$. So in the sequel without loss of generality we will always assume that $N=n$.

2000 Mathematics Subject Classification: Primary 35S30, 35L30; Secondary 58J40.

The paper is in final form and no version of it will be published elsewhere. 
Let $X$ and $Y$ be open sets in $\mathbf{R}^{n}$. A smooth function $a \in C^{\infty}\left(X \times Y \times \mathbf{R}^{n}\right)$ is a symbol of order $m$ and type $\rho, 1 / 2 \leq \rho \leq 1$, if for every compact subset $K$ of $X \times Y$, and any multi-indices $\alpha, \beta$, the estimate

$$
\left|\partial_{x, y}^{\beta} \partial_{\theta}^{\alpha} a(x, y, \theta)\right| \leq C(\alpha, \beta, K)(1+|\theta|)^{m-\rho|\alpha|+(1-\rho)|\beta|}
$$

holds for all $(x, y) \in K$ and $\theta \in \mathbf{R}^{n}$. The space of such symbols is denoted by $S_{\rho, 1-\rho}^{m}(X \times$ $Y \times \mathbf{R}^{n}$ ). If $\rho=1$, we will write $S^{m}$ for $S_{1,0}^{m}$, the space of smooth functions satisfying

$$
\left|\partial_{x, y}^{\beta} \partial_{\theta}^{\alpha} a(x, y, \theta)\right| \leq C(\alpha, \beta, K)(1+|\theta|)^{m-|\alpha|},
$$

for all $(x, y) \in K$ and $\theta \in \mathbf{R}^{n}$.

Fourier integral operators of order $m$ and type $(\rho, 1-\rho)$ are operators that can be locally written in the form

$$
T u(x)=\int_{Y} \int_{\mathbf{R}^{n}} e^{i \Phi(x, y, \theta)} a(x, y, \theta) u(y) d \theta d y,
$$

with some regular phase function $\Phi$ of positive type and a symbol $a \in S_{\rho, 1-\rho}^{m}$. The space of such operators is denoted by $I_{\rho, 1-\rho}^{m}$. As before, we will denote $I_{1,0}^{m}$ by $I^{m}$. Condition (iv) that $\operatorname{Im} \Phi \geq 0$ is necessary for the integral (1) to make sense.

If the phase function $\Phi$ is real valued, one defines the canonical relation $C$ in a neighborhood of $(x, y)$ by

$$
C=\left\{\left(x, d_{x} \Phi(x, y, \theta)\right),\left(y,-d_{y} \Phi(x, y, \theta)\right): d_{\theta} \Phi(x, y, \theta)=0\right\} .
$$

The set $C$ is a conic Lagrangian manifold in $T^{*} X \backslash 0 \times T^{*} Y \backslash 0$ equipped with the standard symplectic form $\sigma_{X} \bigoplus-\sigma_{Y}$. By the equivalence of phase function theorem (see, for example, [2]), there is a bijection between Fourier integral operators with a fixed conic Lagrangian manifold $C \subset T^{*} X \backslash 0 \times T^{*} Y \backslash 0$ and the space of symbols. The set $\left.C^{\prime}=\{(x, \xi, y, \eta)\}:((x, \xi),(y,-\eta)) \in C\right\}$ is the wave front set of the integral kernel of $T$.

If the phase function $\Phi$ is complex valued, an almost analytic continuation $\widetilde{C}$ of $C$ will be a positive homogeneous Lagrangian manifold in the almost analytic manifold $T^{*} \tilde{X} \backslash 0 \times T^{*} \tilde{Y} \backslash 0$. This global description will not be crucial for us as we will discuss local properties of Fourier integral operators for which the representation (1) is sufficient. For the details on the analytic continuation and the calculus of operators with complex phases we refer to [8] and [3].

Continuity properties of Fourier integral operators have been under study for a long time. For example, some earlier references on operators with real valued phase functions arising as solutions to wave equations can be found in [1], [10], [11]. Certain convolution and singular integral operators have been studied in [21], [7]. $L^{p}$-estimates for a class of operators with convex characteristics were established in [23]. General estimates have been finally given by Seeger, Sogge, and Stein in [20]. A survey of these properties can be found in [12]. Seeger, Sogge, and Stein came up with a so-called factorization condition which has been extensively analyzed in [16]. This condition was proved to be automatically satisfied in a number of important cases in [14], in particular in applications to strictly hyperbolic equations in $\mathbf{R} \times \mathbf{R}^{3}$. Best possible orders for $L^{p}$-continuity were determined in [15] in terms of the dimension of the singular support of the operator. 
The calculus of operators with complex phases was developed in [8]. Many applications of this theory can be found in [9]. An alternative approach to such operators is in [6]. $L^{2}$-continuity of operators of order zero was established in [9] and in [5] in more generality. An application of this theory to solutions of Cauchy problems for partial differential equations can be found in [26]. Applications to spectral problems have been investigated in [19]. The $L^{p}$-properties of Fourier integral operators with complex phases have been established in [17]. There one can also find applications to non-hyperbolic partial differential operators, oblique derivative problems, etc. Proofs of most theorems of this paper can be found in [17].

The author is grateful to the Engineering and Physical Sciences Research Council, Great Britain, for grant GR/R67583/01.

2. Estimates for Fourier integral operators with complex phases. Our main assumption on the phase function will be the following local graph type condition:

(L) There exists $\tau \in \mathbf{R}$ such that the real valued phase function $\operatorname{Re} \Phi+\tau \operatorname{Im} \Phi$ defines a local graph in $T^{*} X \times T^{*} Y$,

which means that

$$
\operatorname{det} \partial_{x} \partial_{\theta}(\operatorname{Re} \Phi+\tau \operatorname{Im} \Phi) \neq 0, \quad \operatorname{det} \partial_{y} \partial_{\theta}(\operatorname{Re} \Phi+\tau \operatorname{Im} \Phi) \neq 0
$$

in the support of the symbol $a$. It also means that the projections from the canonical relation defined by the phase function $\operatorname{Re} \Phi+\tau \operatorname{Im} \Phi$ to $T^{*} X \backslash 0$ and $T^{*} Y \backslash 0$ are diffeomorphic. Because of the symplectic structure they will be symplectomorphic as well, which implies that any one of these conditions on determinants implies the other one. If $\Phi$ is real valued, (L) is just the standard local graph condition for $T$. Without this condition even $L^{2}$-properties of $T$ will be much more complicated.

Theorem 1. Let a Fourier integral operator $T \in I^{m}$ have the form (1) with a regular phase function $\Phi$ of positive type satisfying condition $(\mathrm{L})$, and a symbol $a \in S^{m}$. Let $1<p<\infty$. Then $T$ is continuous from $L_{\text {comp }}^{p}(Y)$ to $L_{\mathrm{loc}}^{p}(X)$, provided that $m \leq-(n-1)|1 / p-1 / 2|$.

The proof is based on the complex interpolation between the $L^{2}$-boundedness of operators of order zero and the boundedness of operators of order $-(n-1) / 2$ from the Hardy space $H^{1}$ to $L^{1}$. The $L^{2}$-boundedness of operators from $I^{0}$ follows from the fact that operators from $I^{0}$ with complex phases are also operators with a real phase of order zero and type $(1 / 2,1 / 2)$, ([8], [17]). The latter are locally bounded in $L^{2}([4])$. Proof of Theorem 1 for real phase functions has appeared in [20]. The general case of complex phases was treated in [17].

Properties of operators in $L^{p}$-spaces imply properties in other function spaces as well. First, one can derive $L^{p}-L^{q}$ result for $1<p \leq q \leq 2$ and $2 \leq p \leq q<\infty$.

TheOREm 2. Let a Fourier integral operator $T \in I^{m}$ have the form (1) with a regular phase function $\Phi$ of positive type satisfying condition $(\mathrm{L})$, and a symbol $a \in S^{m}$. Let $1<p \leq q \leq 2$. Then $T$ is continuous from $L_{\text {comp }}^{p}(Y)$ to $L_{\text {loc }}^{q}(X)$, provided that $m \leq 1 / q-n / p+(n-1) / 2$. The dual statement holds for $2 \leq p \leq q<\infty$. 
The proof of this theorem is the complex interpolation between the $L^{2}$-boundedness of operators of order zero, the boundedness of operators of order $-(n-1) / 2$ from the Hardy space $H^{1}$ to $L^{1}$, and the boundedness of operators of order $-n / 2$ from the Hardy space $H^{1}$ to $L^{2}$. For the latter we write $T \in I^{-n / 2}$ as

$$
T=S \circ(I-\Delta)^{-n / 4}, \quad S=T \circ(I-\Delta)^{n / 4} .
$$

Now, $S \in I^{0}$ is bounded from $L^{2}$ to $L^{2}$, and $(I-\Delta)^{-n / 4}$ is bounded from $H^{1}$ to $L^{2}$ by the Hardy space version of the Hardy-Littlewood-Sobolev theorem ([22]).

TheOREM 3. Let a Fourier integral operator $T \in I^{0}$ have the form (1) with a regular phase function $\Phi$ of positive type satisfying condition (L) and a symbol a $\in S^{m}$. Let $1<p<\infty$ and $\alpha \in \mathbf{R}$.

Then $T$ is continuous from the Sobolev space $\left(L_{\alpha+(n-1)|1 / p-1 / 2|}^{p}\right)_{\mathrm{comp}}(Y)$ to the space $\left(L_{\alpha}^{q}\right)_{\text {loc }}(X)$. It is also bounded from the Lipschitz space $\operatorname{Lip}(\alpha+(n-1) / 2)_{\text {comp }}(Y)$ to $\operatorname{Lip}(\alpha)_{\operatorname{loc}}(X)$.

This theorem follows from properties of pseudo-differential operators and Lipschitz spaces. Finally we note that $L^{p}-L^{q}$ estimates with $p \leq 2 \leq q$ are quite different in nature and we do not discuss them here. Let us now state briefly several results for real valued phases. Operator $T \in I^{m}$ is continuous from $L_{\text {comp }}^{p}\left(\mathbf{R}^{n}\right)$ to $L_{\text {loc }}^{q}\left(\mathbf{R}^{n}\right)$ when $m \leq n(1 / q-1 / p)$ and $p \leq 2 \leq q$. Also, when the rank of $\partial_{\theta \theta}^{2} \Phi$ equals $n-1$, this order can be improved. For $1<p, q<\infty, T$ is continuous from $L_{\text {comp }}^{p}\left(\mathbf{R}^{n}\right)$ to $L_{\text {loc }}^{q}\left(\mathbf{R}^{n}\right)$ when $m \leq 1 / q-n / p+(n-1) / 2$ if $q \leq p^{\prime}$, and when $m \leq n / q-1 / p-(n-1) / 2$ if $q \geq p^{\prime}$. Here $p^{\prime}=p /(p-1)$ is the conjugate index to $p$. We refer to $[22, \mathrm{IX}]$ for details. Various geometric conditions for $L^{p}-L^{q}$ continuity of Fourier multipliers have been studied in [24], [25].

3. Estimates for solutions to partial differential equations. Let $X$ be a smooth $n$-dimensional manifold. We consider the Cauchy problem

$$
\begin{cases}P u=0, & t \in[0, T], \\ \partial_{t}^{j} u(0, x)=f_{j}(x), & 0 \leq j \leq m-1,\end{cases}
$$

for an operator $P\left(t, x, \partial_{t}, \partial_{x}\right),(t, x) \in[0, T] \times X$, and $u=u(t, x)$. As usual, let us write $D_{t}=-i \partial_{t}, D_{x}=-i \partial_{x}$. Let $P$ be a differential-pseudo-differential operator on $[0, T] \times X$ of order $m$ of the form

$$
P=D_{t}^{m}+\sum_{l=1}^{m} P_{l}(t) D_{t}^{m-l},
$$

where $P_{l}(t)=P_{l}\left(t, x, D_{x}\right)$ are classical pseudo-differential operators of order $l$ on $X$, depending smoothly on $t$.

It is well known that if $P$ is strictly hyperbolic, then the Cauchy problem (2) is $H^{s}$-well posed. The strict hyperbolicity means that the principal symbol $p(t, x, \tau, \xi)$ of $P\left(t, x, D_{t}, D_{x}\right)$, i.e. the function

$$
p(t, x, \tau, \xi)=\tau^{m}+\sum_{l=1}^{m} p_{l}(t, x, \xi) \tau^{m-l},
$$

has $m$ real distinct roots in $\tau$, where $p_{l}(t, x, \xi)$ are the principal symbols of $P_{l}$, positive homogeneous of degree $l$ in $\xi$ functions, smooth in $[0, T] \times\left(T^{*} X \backslash 0\right)$. 
Now we will consider a more general situation allowing the roots in $\tau$ to be complex valued. We will make the following assumptions:

(A1) $P$ has simple characteristics: for any $(x, \xi) \in T^{*} X \backslash 0$ and any $t \in[0, T]$, the roots $\tau_{j}=\tau_{j}(t, x, \xi)$ of the polynomial $p(t, x, \tau, \xi)$ in $\tau$ are distinct, $j=1, \ldots, m$.

(A2) $\operatorname{Im} \tau_{j}(t, x, \xi) \geq 0$ for all $(t, x, \xi) \in[0, T] \times\left(T^{*} X \backslash 0\right), j=1, \ldots, m$.

It was proved in [26] that under assumptions (A1) and (A2) the Cauchy problem (2) for $P$ is well posed. We will not go into detail of what it includes, but for us it is important that solutions exist and can be represented as a finite sum of Fourier integral operators with complex phases modulo a smooth error. Such smooth error does not play any role in the study of local $L^{p}$-properties of solutions. As a consequence of Theorems 1-3, we obtain local $L^{p}$-properties of solutions.

TheOrem 4. Let $P=P\left(t, x, D_{t}, D_{x}\right)$ be a classical pseudo-differential operator of order $m$ of the form (3). Assume that $P$ satisfies properties (A1), (A2). Let $1<p<\infty$ and $\alpha \in \mathbf{R}$. Let the Cauchy data $f_{j}$ be compactly supported and $f_{j} \in L_{\alpha+(n-1)|1 / p-1 / 2|-j}^{p}$, for all $0 \leq j \leq m-1$. Then for each fixed $t \in[0, T]$ the solution $u=u(t, x)$ to the Cauchy problem (2) satisfies $u(t, \cdot) \in\left(L_{\alpha}^{p}\right)_{\text {loc }}$.

Theorem 5. Let $P$ be as in Theorem 4. Let $1<p \leq q \leq 2$ and $\alpha \in \mathbf{R}$. Let the Cauchy data $f_{j}$ be compactly supported and $f_{j} \in L_{\alpha+1 / q-n / p+(n-1) / 2-j}^{p}$, for all $0 \leq j \leq m-1$. Then for each fixed $t \in[0, T]$ the solution $u=u(t, x)$ to the Cauchy problem (2) satisfies $u(t, \cdot) \in\left(L_{\alpha}^{p}\right)_{\text {loc }}$. The dual statement holds for $2 \leq p \leq q<\infty$. Also, if $f_{j} \in \operatorname{Lip}(\alpha+(n-1) / 2-j)_{\text {comp }}$, then $u(t, \cdot) \in \operatorname{Lip}(\alpha)_{\text {loc }}$.

For $p \leq 2 \leq q, L^{p}-L^{q}$ estimates for operators with constant coefficients can be found in [24], [25]. The orders of regularity can be used to determine global time asymptotics for solutions of Cauchy problems. For example, see [13] for second order equations.

4. Factorization conditions. It turns out that orders in Theorems $1-3$ are not sharp in a number of cases. In general, if the phase function $\Phi$ is real valued and the operator $T$ is elliptic, the orders are sharp, provided that the rank of $\partial_{\theta \theta}^{2} \Phi(x, y, \theta)$ is maximal. Because of the homogeneity of $\Phi$ the maximal rank is equal to $n-1$. However, if $\operatorname{rank} \partial_{\theta \theta}^{2} \Phi(x, y, \theta) \leq k$ in the support of $a$, for some $k, 0 \leq k \leq n-1$, the optimal orders for the $L^{p}$-boundedness depend on $k$. If $k=0$, it is shown in [15] that by a change of variables $T$ becomes a pseudo-differential operator. In this case $T \in I^{0}$ is bounded from $L_{\text {comp }}^{p}$ to $L_{\text {loc }}^{p}$, i.e. there is no loss of smoothness in $L^{p}$-spaces for all $1<p<\infty$. An important ingredient to determine the orders is the following factorization type condition:

(F) Let condition (L) hold with some real $\tau,|\tau|<1 / \sqrt{3}$. Let $k, 0 \leq k \leq n-1$, be such that

$$
\operatorname{rank} \partial_{\theta \theta}^{2}(\operatorname{Re} \Phi(x, y, \theta)+\tau \operatorname{Im} \Phi(x, y, \theta)) \leq k
$$

in the support of $a$, with the equality for $(x, y, \theta)$ in a set $\Omega \neq \emptyset$. We assume now that the mapping

$$
\operatorname{ker}(\operatorname{Re} \Phi(x, y, \theta)+\tau \operatorname{Im} \Phi(x, y, \theta))
$$


can be smoothly extended from $\Omega$ to the support of $a$ as a mapping to the Grassmanian of $(n-k)$-dimensional linear subspaces of $\mathbf{R}^{n}$.

The geometric meaning of this condition can be explained as follows. Let $\Psi=\operatorname{Re} \Phi+$ $\tau \operatorname{Im} \Phi$ with some real $\tau,|\tau|<1 / \sqrt{3}$, for which $\Psi$ defines a local graph, i.e. condition (L) is satisfied. Then condition $(\mathrm{F})$ is equivalent to the smooth factorization condition for the real valued phase function $\Psi$, introduced in [20]. Namely, let

$$
\Lambda_{\Psi}=\left\{\left(x, y, d_{x} \Psi(x, y, \theta), d_{y} \Psi(x, y, \theta)\right): d_{\theta} \Psi(x, y, \theta)=0\right\} \subset T^{*}(X \times Y) .
$$

Then condition (F) is equivalent to saying that for every $\lambda \in \Lambda_{\Psi}$, there exists a homogeneous of zero order map $\pi_{\lambda}$, smooth in a neighborhood of $\lambda$ in $\Lambda_{\Psi}$, of constant rank, $\operatorname{rank} d \pi_{\lambda}=n+k$, such that

$$
\left.\pi_{X \times Y}\right|_{\Lambda_{\Psi}}=\left.\pi_{X \times Y}\right|_{\Lambda_{\Psi}} \circ \pi_{\lambda},
$$

in a neighborhood of $\lambda$. Here $\pi_{X \times Y}$ is the canonical projection from $T^{*}(X \times Y)$ to $X \times Y$. The mapping defined by $\operatorname{ker} \pi_{\lambda}$ corresponds to the extension of $\operatorname{ker}(\operatorname{Re} \Phi(x, y, \theta)+$ $\tau \operatorname{Im} \Phi(x, y, \theta))$ from condition $(\mathrm{F})$, lifted to $\Lambda_{\Psi}$. This equivalence is discussed in [17] in detail.

Note that condition (F) holds trivially when $k=0$ or $k=n-1$. So, in this cases we only have to assume condition (L). Now we will formulate the $L^{p}$-properties of operators under condition $(\mathrm{F})$. For $k=n-1$ this theorem extends Theorem 1 to operators in $I_{\rho, 1-\rho}^{m}$.

TheOREM 6. Let a Fourier integral operator $T \in I_{\rho, 1-\rho}^{m}$ have the form (1) with a regular phase function $\Phi$ of positive type satisfying condition $(\mathrm{F})$ with some $k$, a symbol $a \in S_{\rho, 1-\rho}^{m}$, and $1 / 2 \leq \rho \leq 1$. Let $1<p<\infty$. Then $T$ is continuous from $L_{\text {comp }}^{p}(Y)$ to $L_{\text {loc }}^{p}(X)$, provided that $m \leq-(k+(n-k)(1-\rho))|1 / p-1 / 2|$.

Similar to Theorems 2 and 3 , we get estimates in other spaces.

As we have already mentioned, in some cases the factorization condition ( $\mathrm{F}$ ) holds automatically. In addition to $k=0$ and $k=n-1$, this happens in spaces of small dimensions for operators $T$ commuting with translations. The following two theorems were proved in [17].

ThEOREM 7. Let a Fourier integral operator $T \in I_{\rho, 1-\rho}^{m}$ have the form (1) with a regular phase function $\Phi$ of positive type satisfying condition $(\mathrm{L})$, a symbol a $\in S_{\rho, 1-\rho}^{m}$, and $1 / 2 \leq \rho \leq 1$. Let $1<p<\infty$. Assume that $T$ commutes with translations and that $\Phi$ is a complex valued function which is real analytic in $(x, y, \theta)$ on the support of a. Assume that there exists a real $\tau,|\tau|<1 / \sqrt{3}$, and an integer $k, 0 \leq k \leq 2$, such that

$$
\operatorname{rank} \partial_{\theta \theta}^{2}(\operatorname{Re} \Phi+\tau \operatorname{Im} \Phi) \leq k
$$

in the support of a. Then $T$ is continuous from $L_{\text {comp }}^{p}(Y)$ to $L_{\mathrm{loc}}^{p}(X)$, provided that $m \leq-(k+(n-k)(1-\rho))|1 / p-1 / 2|$.

If the dimension $n \leq 4$, we can allow $k=3$ as well.

THEOREM 8. Let $n \leq 4$. Let a Fourier integral operator $T \in I_{\rho, 1-\rho}^{m}$ have the form (1) with a regular phase function $\Phi$ of positive type satisfying condition (L), a symbol $a \in S_{\rho, 1-\rho}^{m}$, and $1 / 2 \leq \rho \leq 1$. Let $1<p<\infty$. Assume that $T$ commutes with translations and that $\Phi$ is a complex valued function which is real analytic in $(x, y, \theta)$ on the 
support of a. Assume that there exists a real $\tau,|\tau|<1 / \sqrt{3}$, and an integer $k$, such that

$$
\operatorname{rank} \partial_{\theta \theta}^{2}(\operatorname{Re} \Phi+\tau \operatorname{Im} \Phi) \leq k
$$

in the support of a. Then $T$ is continuous from $L_{\text {comp }}^{p}(Y)$ to $L_{\text {loc }}^{p}(X)$, provided that $m \leq-(k+(n-k)(1-\rho))|1 / p-1 / 2|$.

We have similar estimates in other spaces.

THEOREM 9. Let a Fourier integral operator $T \in I^{m}$ satisfy conditions of Theorem 7 or Theorem 8. Let $1<p \leq q \leq 2$. Then $T$ is continuous from $L_{\text {comp }}^{p}(Y)$ to $L_{\text {loc }}^{q}(X)$, provided that $m \leq(n-k) / q-n / p+k / 2$. The dual statement holds for $2 \leq p \leq q<\infty$.

THEOREM 10. Let a Fourier integral operator $T \in I^{0}$ satisfy conditions of Theorem 7 or Theorem 8. Let $1<p<\infty$ and $\alpha \in \mathbf{R}$. Then $T$ is continuous from the Sobolev space $\left(L_{\alpha+k|1 / p-1 / 2|}^{p}\right)_{\text {comp }}(Y)$ to $\left(L_{\alpha}^{q}\right)_{\text {loc }}(X)$. It is also bounded from the Lipschitz space $(\operatorname{Lip}(\alpha+k / 2))_{\text {comp }}(Y)$ to $(\operatorname{Lip}(\alpha))_{\text {loc }}(X)$.

5. Further estimates. An important application of Theorem 8 is the estimate for the solutions of the Cauchy problem (2) in $\mathbf{R} \times \mathbf{R}^{n}$ with $n \leq 4$. In this case it was shown in [17] that the factorization type condition $(\mathrm{F})$ is satisfied and we get

THEOREM 11. Let $P$ be a classical pseudo-differential operator with analytic (in $\xi$ ) symbol of order $m$ of the form (3) in $\mathbf{R} \times \mathbf{R}^{n}, n \leq 4$. Assume that coefficients $P_{j}$ of $P$ may depend on time, but are constant in $x$. Assume that $P$ has simple characteristics $\tau_{j}=\tau_{j}(t, \xi), j=1, \ldots, m$, that satisfy

$$
\operatorname{Im} \tau_{j}(t, \xi) \geq 0
$$

for all $\xi$ and $t \in[0, T]$. Assume that

$$
\operatorname{rank} \partial_{\xi \xi}^{2} \tau_{j}(t, \xi) \leq k
$$

for all $\xi, t \in[0, T]$, and $1 \leq j \leq m$. Let $1<p<\infty, \alpha \in \mathbf{R}$. Let the Cauchy data $f_{j}$ be compactly supported and $f_{j} \in L_{\alpha+k|1 / p-1 / 2|-j}^{p}, 0 \leq j \leq m-1$. Then for all fixed $t \in[0, T]$ we have $u(t, \cdot) \in\left(L_{\alpha}^{p}\right)_{\text {loc }}$. We also have the estimate

$$
\|u(t, \cdot)\|_{L_{\alpha}^{p}} \leq C_{T} \sum_{j=0}^{m-1}\left\|f_{j}\right\|_{L_{\alpha+k|1 / p-1 / 2|-j}^{p}}, \quad \forall t \in[0, T] .
$$

Similar estimates hold in Sobolev $L^{q}$ - and Lipschitz spaces.

The $L^{p}$-estimates in this paper are in general sharp. In fact, if the phase function is real valued and the symbol is elliptic, then the loss of $(n-1)|1 / p-1 / 2|$ derivatives in $L^{p}$ is sharp when the singular support of the Fourier integral operator has the highest dimension $n-1$ at some point. This corresponds to the rank of $\partial_{\theta \theta}^{2}(\operatorname{Re} \Phi+\tau \operatorname{Im} \Phi)$ being equal to $n-1$ at some point. However, Theorems 8-9 show that if the factorization condition (F) holds for $\operatorname{Re} \Phi+\tau \operatorname{Im} \Phi$, and the rank of its Hessian is less than or equal to $k$, then there is a loss of only $k|1 / p-1 / 2|$ derivatives. In particular, if $k=0$, there is no loss of smoothness in $L^{p}$.

It was shown in [15] that if the rank of this Hessian is equal to $k$ at some point and the symbol is elliptic, then there must be a loss of at least $k|1 / p-1 / 2|$ derivatives 
in $L^{p}$. Hence in Theorem 8 under the factorization condition (F) the loss of $k|1 / p-1 / 2|$ derivatives is sharp.

It is natural to investigate what happens if the factorization condition $(F)$ fails. Let us now give an example of [18].

ThEOREM 12. Let $T \in I^{m}$ be a Fourier integral operator in $\mathbf{R}^{3}$ with symbol $a \in S^{m}$ and the real valued phase function

$$
\Phi(x, y, \theta)=\langle x-y, \theta\rangle-\frac{1}{\theta_{3}}\left(y_{1} \theta_{1}+y_{2} \theta_{2}\right)^{2},
$$

microlocally in a cone $\left|\left(\theta_{1}, \theta_{2}\right)\right| \leq C\left|\theta_{3}\right|$ for some $C>0$. Let $1<p<\infty$. Then the factorization condition $(\mathrm{F})$ fails, but $T$ is still continuous from $L_{\text {comp }}^{p}\left(\mathbf{R}^{3}\right)$ to $L_{\mathrm{loc}}^{p}\left(\mathbf{R}^{3}\right)$, provided that $m \leq-|1 / p-1 / 2|$.

Since the rank of the Hessian of $\Phi$ does not exceed $k=1$, according to [15] one has to lose at least $|1 / p-1 / 2|$ derivatives whenever $a$ is elliptic. Theorem 12 shows that one loses exactly $|1 / p-1 / 2|$ derivatives even though the factorization condition $(\mathrm{F})$ fails. Similar examples are readily generalized to other values of $n$ and $k$.

6. The oblique derivative problem. Let $X$ be a compact $n$-dimensional manifold and let $\Gamma$ be a smooth compact hypersurface in $X$. Assume that $X \backslash \Gamma$ is the union of two disjoint open sets $X_{+}$and $X_{-}$, having $\Gamma$ as their common boundary.

Let $S_{c}^{m}$ be the set of classical symbols of order $m: a \in S_{c}^{m}$ if $a \in S_{1,0}^{m}$ and

$$
a(x, \theta) \sim \sum_{j=0}^{\infty} a_{m-j}(x, \theta)
$$

is a representation as a convergent asymptotic series locally in all open conic subsets of $T^{*} X \backslash 0$, with all $a_{m-j}$ being positively homogeneous of degree $m-j$ in $\theta$.

Let $P \in \Psi_{c}^{1}(X)$ be a classical pseudo-differential operator on $X$ of order one of the form

$$
P\left(x, D_{x}\right)=m\left(x, D_{x}\right)+i Q\left(x, D_{x}\right),
$$

where $m\left(x, D_{x}\right)$ is a real vector field on $X$ and $Q \in \Psi_{c}^{1}(X)$ has a real principal symbol $q=q(x, \xi)$. We assume that $q$ changes its sign on $\Gamma$ :

$$
q(x, \xi) \leq 0 \text { for } x \in X_{-}, \quad q(x, \xi) \geq 0 \text { for } x \in X_{+} .
$$

Let $K \subset X$ be the set of all points $x \in X$ such that $q(x, \xi)=0$ for some $\xi \neq 0$. This set $K$ contains $\Gamma$ because of inequalities (4) and smoothness of $q$.

We will assume that $m$ does not vanish on $K$, that no maximal curve of the vector field $m$ is entirely contained in $K$, and that $m$ is transversal to $\Gamma$ and points into $X_{+}$.

Outside of characteristics of $P$, a microlocal parametrix for $P$ exists and is unique. Let $P^{-1}$ be the right parametrix for $P$. This parametrix was constructed in [9] and it was shown that $P \circ P^{-1} \equiv I$ and $P^{-1} \circ P=I-F$, where $F$ is a Fourier integral operator of order zero with a complex valued phase function. It was shown in [9] that $P^{-1}$ is bounded from $\left(L_{\alpha}^{2}\right)_{\text {comp }}\left(X_{ \pm}\right)$to $L_{\alpha}^{2}(X)$ and from $L_{\alpha}^{2}(X)$ to $L_{\alpha}^{2}(X)$, for all $\alpha$. It turns out that this parametrix $P^{-1}$ is a Fourier integral operator with complex phase and our estimates of Theorem 1 extend the $L^{2}$ estimates to $L^{p}$ spaces. 
TheOrem 13. Let $1<p<\infty, \alpha \in \mathbf{R}$ and $q \geq 2$. Then $P^{-1}$ is continuous from the space $\left(L_{\alpha+(n-1)|1 / p-1 / 2|}^{p}\right)_{\text {comp }}\left(X_{ \pm}\right)$to $\left(L_{\alpha}^{p}\right)_{\mathrm{loc}}(X)$ and from $\left(L_{\alpha+n(1 / 2-1 / q)}^{2}\right)_{\mathrm{comp}}(X)$ to $\left(L_{\alpha}^{q}\right)_{\mathrm{loc}}(X)$.

\section{References}

[1] M. Beals, $L^{p}$ boundedness of Fourier integrals, Mem. Amer. Math. Soc. 264 (1982).

[2] J. J. Duistermant, Fourier Integral Operators, Progr. Math. 130, Birkhäuser, Boston, 1996.

[3] Yu. V. Egorov, Microlocal analysis, in: Partial Differential Equations IV, Encyclopaedia Math. Sci. 33, Springer, Berlin, 1993, 1-147.

[4] A. Greenleaf and G. Uhlmann, Estimates for singular Radon transforms and pseudodifferential operators with singular symbols, J. Funct. Anal. 89 (1990), 202-232.

[5] L. HöRmANDER, $L^{2}$ estimates for Fourier integral operators with complex phase, Ark. Mat. 21 (1983), 283-307.

[6] L. Hörmander, The Analysis of Linear Partial Differential Operators, vols. III-IV, Springer, Berlin, 1985.

[7] W. Littman, $L^{p}-L^{q}$-estimates for singular integral operators arising from hyperbolic equations, in: Partial Differential Equations (Berkeley, 1971), Proc. Symp. Pure Math. 23, Amer. Math. Soc., Providence, 1973, 479-481.

[8] A. Melin and J. SJÖstrand, Fourier integral operators with complex-valued phase functions, in: Fourier Integral Operators and Partial Differential Equations (Nice, 1974), Lecture Notes in Math. 459, Springer, Berlin, 1975, 120-223.

[9] A. Melin and J. SJöstrand, Fourier integral operators with complex phase functions and parametrix for an interior boundary problem, Comm. Partial Differential Equations 1 (1976), 313-400.

[10] A. MiYACHI, On some estimates for the wave operator in $L^{p}$ and $H^{p}$, J. Fac. Sci. Univ. Tokyo Sect. IA Math. 27 (1980), 331-354.

[11] J. Peral, $L^{p}$ estimates for the wave equation, J. Funct. Anal. 36 (1980), 114-145.

[12] D. H. Phong, Regularity of Fourier integral operators, in: Proceedings of the International Congress of Mathematicians (Zürich, 1994), vol. 1-2, Birkhäuser, Basel, 1995, 862-874.

[13] M. Reissig and K. Yagdjian, $L_{p}-L_{q}$ decay estimates for the solutions of strictly hyperbolic equations of second order with increasing in time coefficients, Math. Nachr. 214 (2000), 71-104.

[14] M. Ruzhansky, Analytic Fourier integral operators, Monge-Ampère equation and holomorphic factorization, Arch. Mat. (Basel) 72 (1999), 68-76.

[15] M. Ruzhansky, On the sharpness of Seeger-Sogge-Stein orders, Hokkaido Math. J. 28 (1999), 357-362.

[16] M. V. Ruzhansky, Singularities of affine fibrations in the theory of regularity of Fourier integral operators, Uspekhi Mat. Nauk 55 (2000), no. 1, 99-170 (in Russian); English transl.: Russian Math. Surveys 55 (2000), 93-161.

[17] M. Ruzhansky, Regularity Theory of Fourier Integral Operators with Complex Phases and Singularities of Affine Fibrations, CWI Tract 131, Stichting Mathematisch Centrum, Centrum voor Wiskunde en Informatica, Amsterdam, 2001.

[18] M. Ruzhansky, On the failure of the factorization condition for non-degenerate Fourier integral operators, Proc. Amer. Math. Soc. 130 (2002), 1371-1376.

[19] Yu. Safarov and D. Vassiliev, The Asymptotic Distribution of Eigenvalues of Partial Differential Operators, Transl. Math. Monogr. 155, Amer. Math. Soc., Providence, 1996. 
[20] A. Seeger, C. D. Sogge and E. M. Stein, Regularity properties of Fourier integral operators, Ann. of Math. (2) 134 (1991), 231-251.

[21] E. M. Stein, $L^{p}$ boundedness of certain convolution operators, Bull. Amer. Math. Soc. 77 (1971), 404-405.

[22] E. M. Stein, Harmonic Analysis: Real-Variable Methods, Orthogonality, and Oscillatory Integrals, Princeton Math. Ser. 43, Princeton University Press, Princeton, 1993.

[23] M. Sugimoto, On some $L^{p}$-estimates for hyperbolic equations, Ark. Mat. 30 (1992), 149-163.

[24] M. Sugimoto, A priori estimates for higher order hyperbolic equations, Math. Z. 215 (1994), $519-531$.

[25] M. Sugimoto, Estimates for hyperbolic equations with non-convex characteristics, Math. Z. 222 (1996), 521-531.

[26] F. TRÈves, Introduction to Pseudodifferential and Fourier Integral Operators, vol. 2: Fourier Integral Operators, Plenum Press, London, 1980. 